\title{
Die Umkehrungsformen des Additiven im Poly-Werk
}

Um ein umfassendes Verständnis zum Thema zu entwickeln, verfolge ich hier einen gemischten Forschungsansatz, der auch das künstlerische Forschungspotenzial miteinfließen lässt. Indem Forschung selbst kreativ wird und künstlerische Experimente wagt, sollen neue Handlungs- und Erfahrungsräume erschlossen werden. Ein rein musiktheoretischer oder musikwissenschaftlicher Forschungsansatz würde die Theoriebildung ausschließlich auf eine vergangene Entwicklung stützen und bei diesem Thema Gefahr laufen, das Poly-Werk als rein expandierende Werkform aufzufassen, da das Gros der Werkzyklen in ihrer Entstehungsrichtung additiv auf Zuwachs und Vergrößerung angelegt ist. Außerdem wäre der kompositorische Prozess, der neuerdings auch die Gestaltung des interformalen Raums zwischen den Werken umfasst und als ein „Gestalten in der Differenz" beschrieben wurde, nicht sichtbar. Aus diesem Grund habe ich eigene Kompositionen entwickelt, die sowohl parallel zum als auch in Verschränkung mit dem wissenschaftlichen und analytischen Teil entstanden.

Grundsätzlich hat sich das Poly-Werk in den vergangenen Jahrzehnten tatsächlich als eine kumulative Werkform weiterentwickelt, die das Verhältnis zwischen verschiedenen individuellen Werken und Werkgruppen verschiedener Größe vielfältig und teilweise komplex modelliert. Im Vergleich zu den sich ebenso einseitig vergrößernden Werkerweiterungen und Simultanaufführungen, die vor allem das „quantitative Mehr“ beschwören, wurde durch die Verzahnung der Werke im (prä-)kompositorischen Prozess bereits eine Wende zu einem „qualitativen Plus“ vollzogen. Dennoch verdeckt diese Entwicklung ein weiteres Potenzial des Poly-Werks, das in seinem Kern eigentlich keine additive, sondern eine extrem ambigue Werkform ist, die ebenso auch andere Entstehungsrichtungen - wie beispielsweise Reduktion, Subtraktion, Spaltung oder Kreuzung - verfolgen kann. Diese weiteren denkbaren Differenzierungen sind im Vergleich allerdings viel weniger verfolgt worden oder oft nicht eindeutig zuzuordnen, da Additivität und Subtraktivität in vielen Fällen zwei Seiten einer Medaille sind. Dazu kommt, dass - je nach Hörerfahrung - auch unterschiedlich wahrgenommen werden kann, ob etwas als 
ursprüngliche Zusammenschließung oder als tatsächliche Splittung konzipiert war. Bereits diese Ambiguität konstituiert einen kreativen Rahmen, innerhalb dessen weitere Synthese-, Anwendungs- und Wandlungsmöglichkeiten von Ganzheit im Poly-Werk ausgelotet werden können.

\subsection{Zwischen Dividuum und Dyade - Die Erschließung des Poly-Werks aus einer performativen Perspektive}

Als Ganzheit ist das Poly-Werk nie eindeutig, da jede Ganzheit sowohl in sich selbst als - teilbare oder unteilbare - Ganzheit existiert als auch Teil einer größeren Ganzheit sein kann. Die kleinste unteilbare Einheit ist immer ein in sich geschlossenes Werk. Als kleinstmögliche, nicht mehr weiter teilbare Ganzheit kommt in der bisherigen Entwicklung des Poly-Werks das Solostück mit einem einzelnen Instrument, gespielt von einem einzelnen Spieler in Betracht. Aus meiner künstlerischen Perspektive war es spannend, gerade diese vermeintliche Untergrenze in den Blick zu nehmen und die bis dahin integrale Instrumentaleinheit - und damit die integrale Solokomposition aufzuspalten. Das im Rahmen des Forschungsprojekts von mir komponierte Violinenstück Dividuum geht beispielsweise diesen Schritt. Die Komposition besteht aus zwei Solostücken für Violine, die sich zu einem neuen dritten Solo verbinden können. Die Aufspaltung der Soloeinheit in der Werkganzheit erweitert den kompositorischen Handlungsraum um eine neue performative Dimension. Denn nicht mehr nur die Werkeinheit ist ambigue, auch der Interpret geht diesen Schritt. Er ist jetzt nicht mehr nur Individuum einerseits oder Teil eines größeren Kollektivs andererseits, sondern der Raum der Differenz verschiebt sich in das Individuum hinein. Diese Möglichkeit eröffnet neue Zugänge zu einer mannigfaltigen Werkmehrung jenseits von instrumentalen Besetzungserweiterungen.

Der im folgenden vorgestellte Zyklus X-Pieces, der im Rahmen des Forschungsprojekts eine Art Labor für die unterschiedlichen Möglichkeiten und Richtungen der Werkkombination darstellte, reduziert die Besetzung auf zwei Soloinstrumente und Spieler, die sowohl einzeln als auch in verschiedenen Formationen zusammenkommen können. Die Duokonstellation bildet als Zweierbeziehung die kleinste Form einer Gruppe und damit einen konzentrierten Versuchsrahmen für die verschiedenen engen oder weiten Formen von Zusammen- und Getrenntheit. 


\subsection{X-Pieces für zwei Blockflöten (2017)}

Der Zyklus X-Pieces für zwei Blockflöten ist ein Zyklus aus mehreren Werken und Fassungen, der auf zwei Solostücken für Blockflöte basiert. Die Solostücke heißen Slash und Backslash. Sie sind die beiden Säulen, die den Zyklus tragen. Abbildung 51 zeigt die ersten fünf Takte in einer simultanen Darstellung.

Die oberen beiden Systeme gehören zum Stück Slash, die unteren beiden Systeme zum Stück Backslash. Die Notation für jedes Stück untergliedert sich in je zwei Systeme, wobei das obere System der Artikulation und das untere System der Grifftechnik zugeordnet ist. Das Tonhöhensystem repräsentiert nicht die eigentlichen Tonhöhen. Es handelt sich um eine reine Griffnotation, da die resultierende Tonhöhe auf der Blockflöte im Zusammenspiel von Griff, Dynamik und Artikulation entsteht. Die Separierung und Modularisierung ermöglicht auch eine Aufteilung der Spielaktionen unter den Spielern.

\section{Addition und Subtraktion}

Ausgehend von den beiden Solostücken existieren verschiedene Richtungen der simultanen Überlagerung (siehe Abb. 52). Die einfachste Form der Simultaneisierung wird durch eine einfach additive Duoversion verkörpert, bei der sich die beiden Solostücke Slash und Backslash als Ganzes begegnen. Diese Version trägt den Titel $V$. Weitere Möglichkeiten der simultanen Kombination ergeben sich aus der Aufspaltung der Artikulations- und der Griffebene, die in den beiden Solostücken von vornherein angelegt war. Beispielsweise können die „Fingerstimmen“ - traditionell gelesen - aus dem Notentext herausgeeinzelt werden und bilden abgelöst von der Artikulationsstimme nun zwei weitere autonome Solostücke aus. Diese Stücke tragen den Werktitel Hidden Piece. Beide Stücke können aber auch wieder miteinander addiert werden zum Duo Hidden $V$.

\section{Kreuzung}

Drei weitere Werkvarianten entstehen aus einer Spielpraxis, die die Stücke und die Körper der Spieler miteinander verschränkt. Die Versionen $N_{1}$ und $N_{2}$ setzen voraus, dass das Mittel- und Kopfstück der Blockflöte um neunzig Grad gegeneinander gedreht werden. Dadurch ergibt sich eine neue Position für die Spieler. Sie macht es möglich, die Artikulationsstimme eines Solos mit der Fingerstimme des anderen Solos zu kombinieren und auf diese Weise das Spiel auf einer Flöte unter den Spielern aufzuteilen (siehe Abb. 53 links). Die Artikulationsebene von Slash in Kombination mit der Griffebene von 

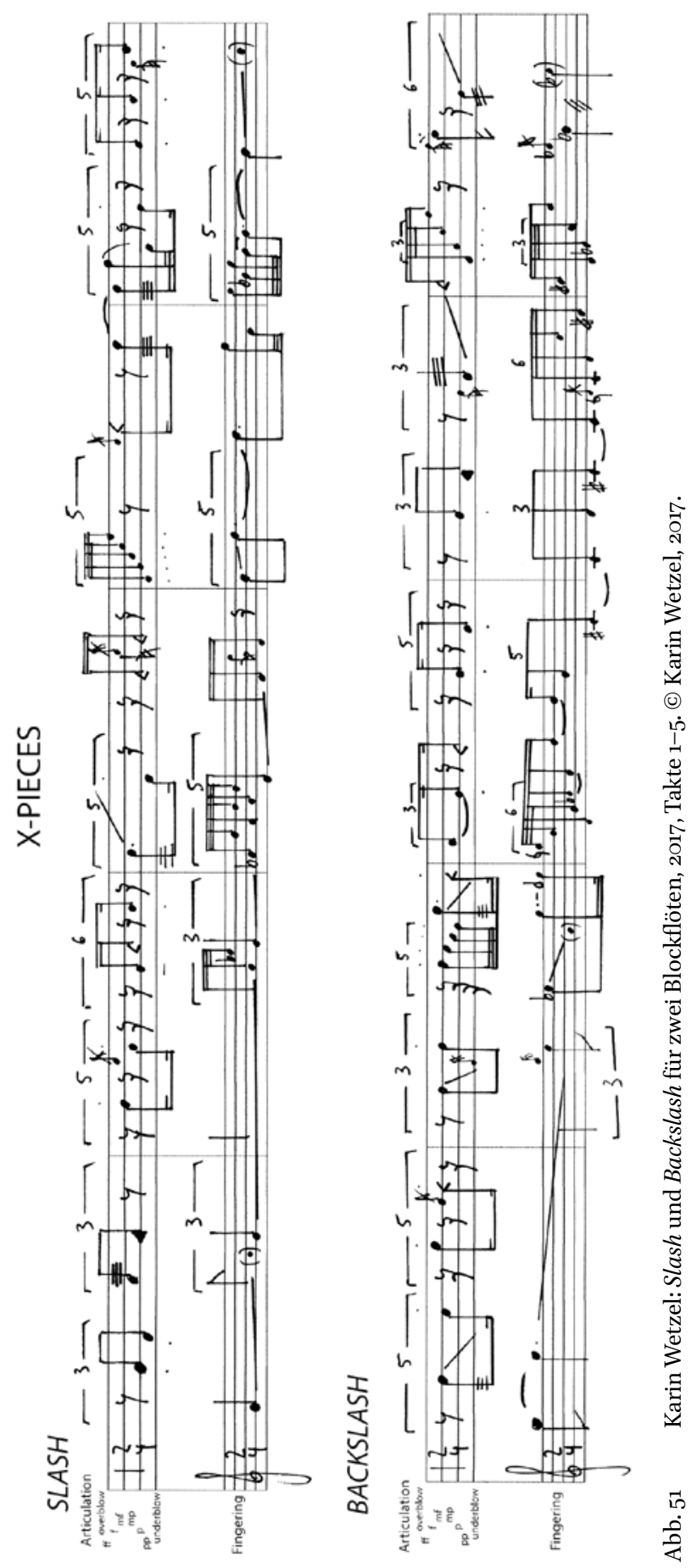


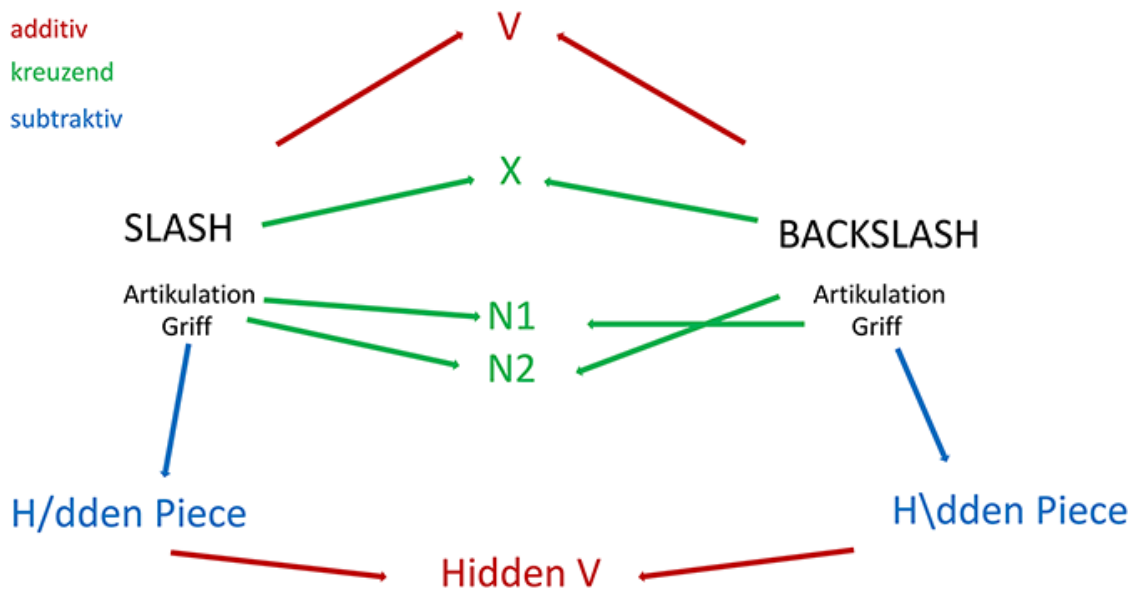

Abb. $5^{2}$ Übersicht möglicher Werkformen in X-Pieces.

Backslash bildet die Version N1. Die Artikulationsebene von Backslash in Kombination mit der Griffebene von Slash entsprechend die Version N2. Beide Versionen stellen ein Duo auf einem Instrument dar.

Für die Aufführung der $X$-Version werden Mund- und Mittelstücke der Instrumente um 180 Grad gegeneinander gedreht. Beide Musiker sitzen sich frontal gegenüber, blasen in die eigene Flöte, greifen aber die Grifflöcher der Flöte des Partners und überkreuzen ihre Flöten ungefähr in Form eines X. In dieser neuen Position verschränken beide ihre Soloversionen (siehe Abb. 53 rechts).
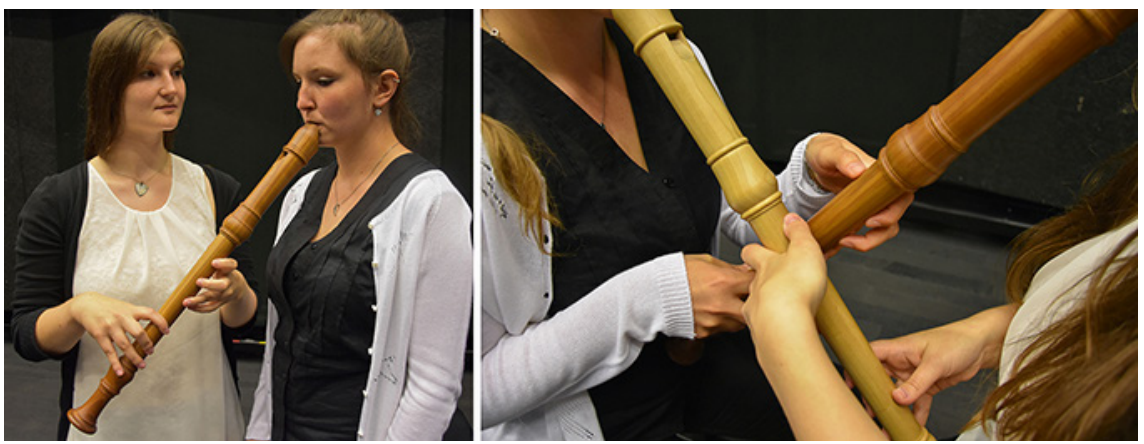

Abb. 53

Dividuales Spiel in $N$ (links), dividuales Spiel in $X$ (rechts). Lydia Dietrich und Laura Kalchofner, Blockflöten. Printed with permission. 


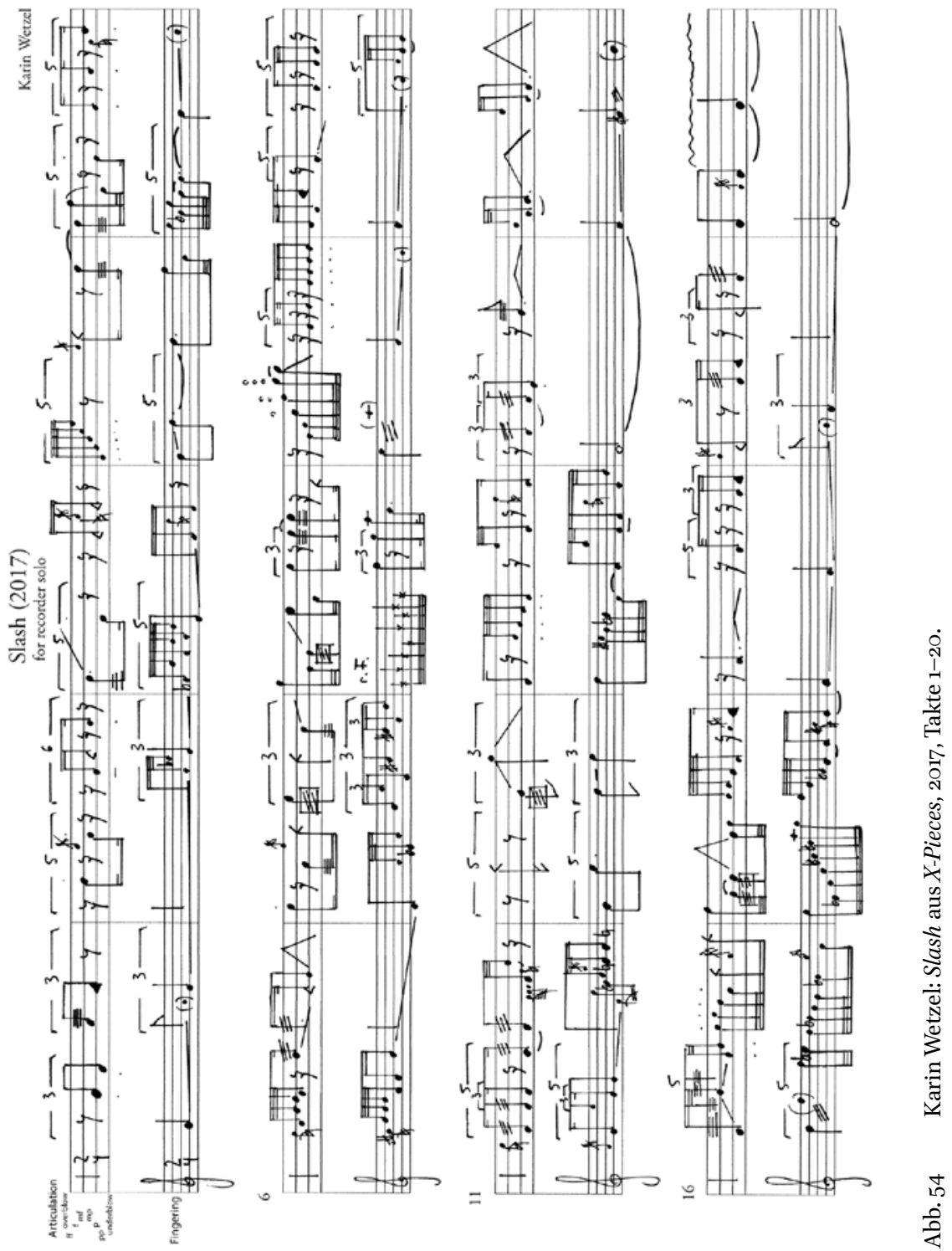




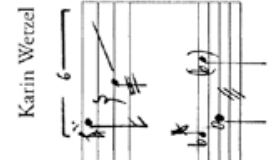

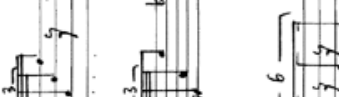

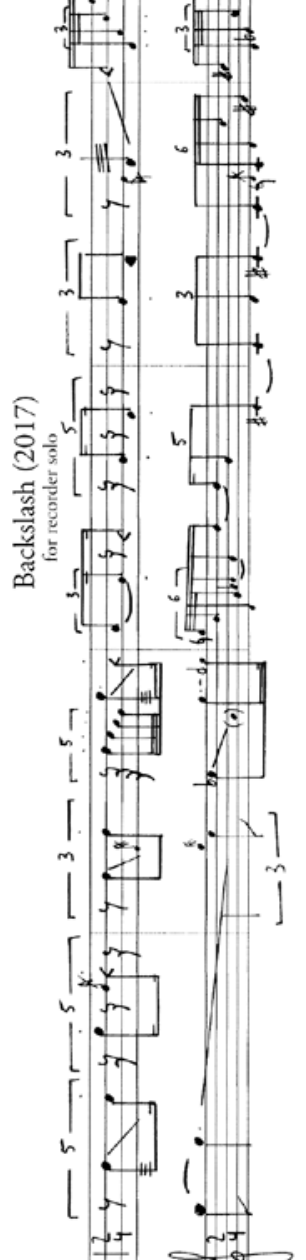

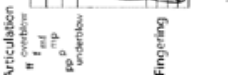


$1 \mathrm{iti}$
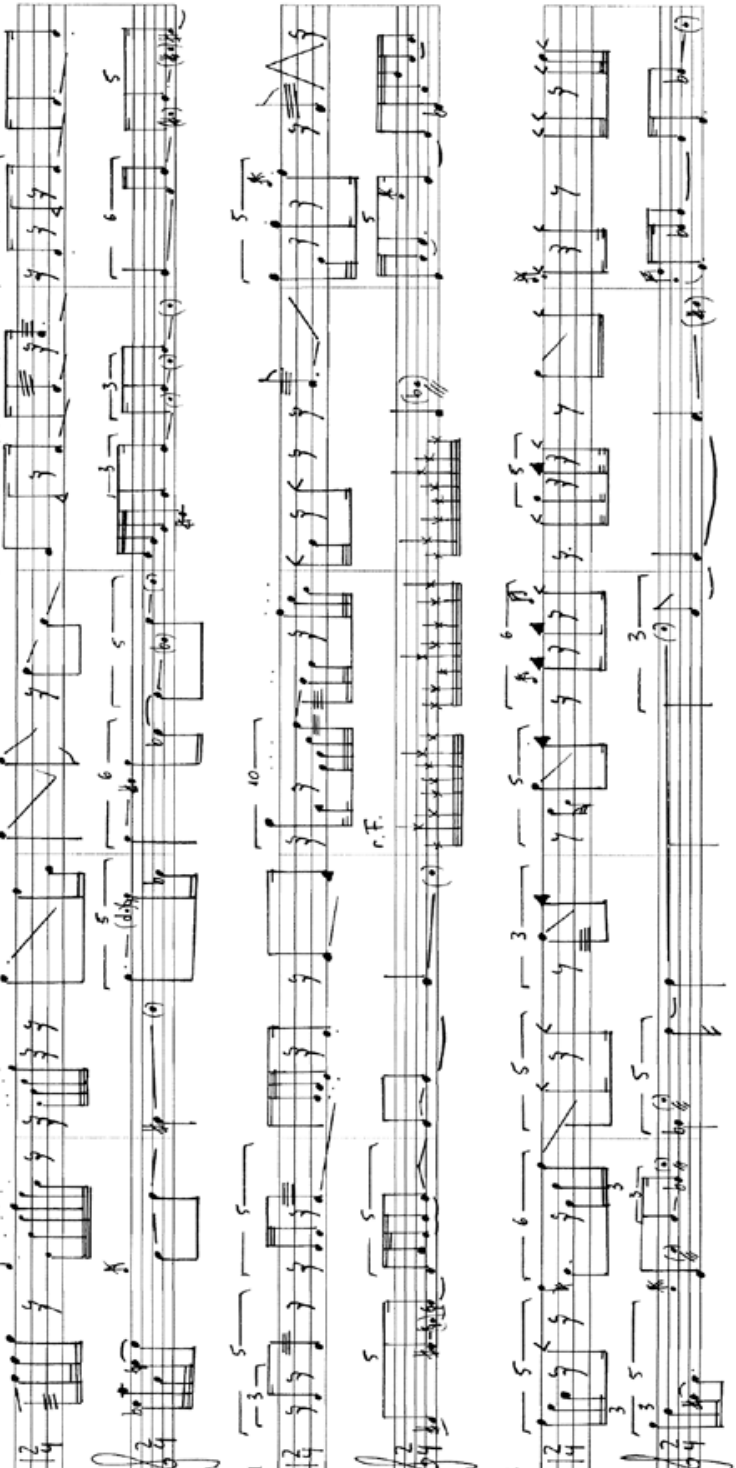

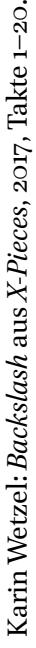

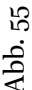



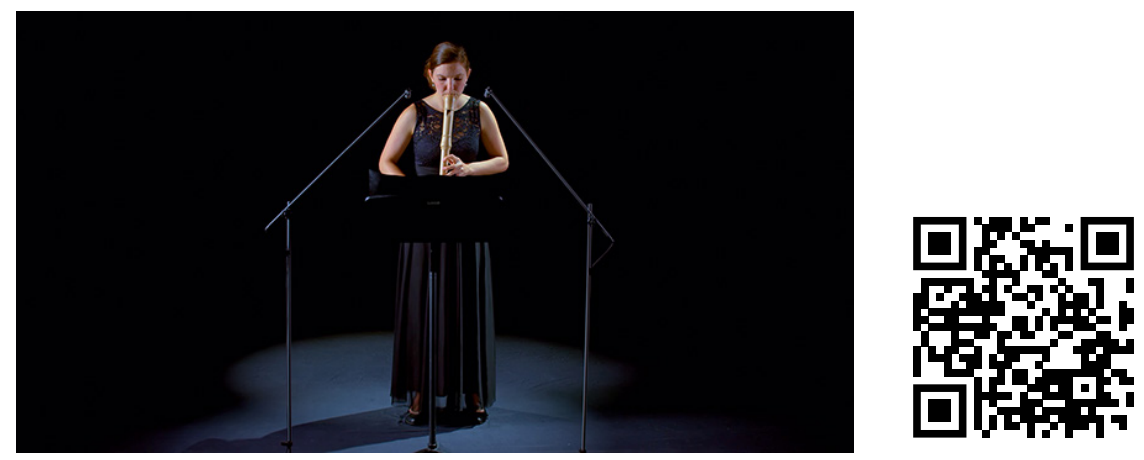

Abb. $5^{6} \quad$ Karin Wetzel: Slash aus X-Pieces für Blockflöte, 2017. Lydia Dietrich, Blockflöte. Printed with permission. Zur Aufnahme: https://vimeo.com/640829212.
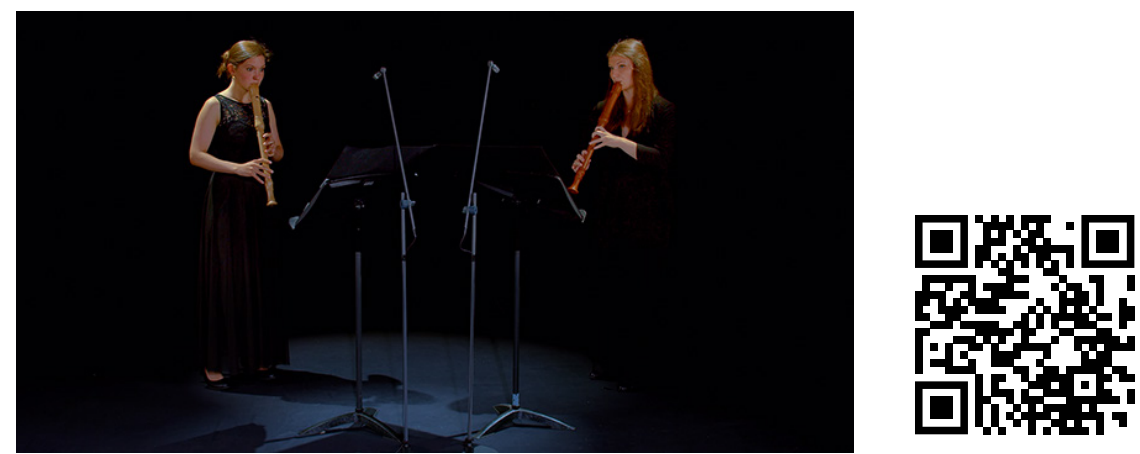

Abb. 57 Karin Wetzel: $V$ aus X-Pieces für zwei Blockflöten, 2017. Lydia Dietrich und Laura Kalchofner, Blockflöten. Printed with permission. Zur Aufnahme: https://vimeo.com/64030436o.
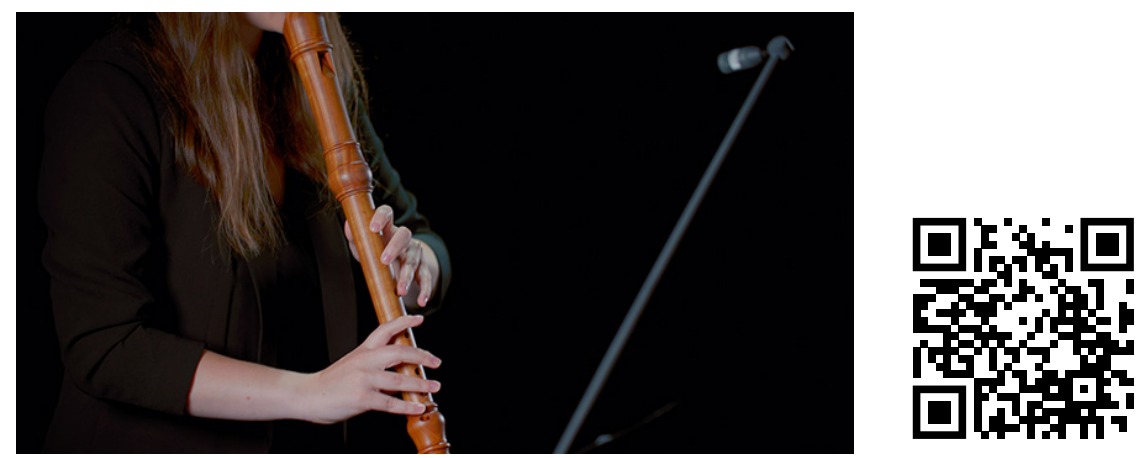

Abb. $5^{8} \quad$ Karin Wetzel: Hidden Piece aus X-Pieces für Blockflöte, 2017. Laura Kalchofner, Blockflöte. Printed with permission.

Zur Aufnahme: https://vimeo.com/640273936. 

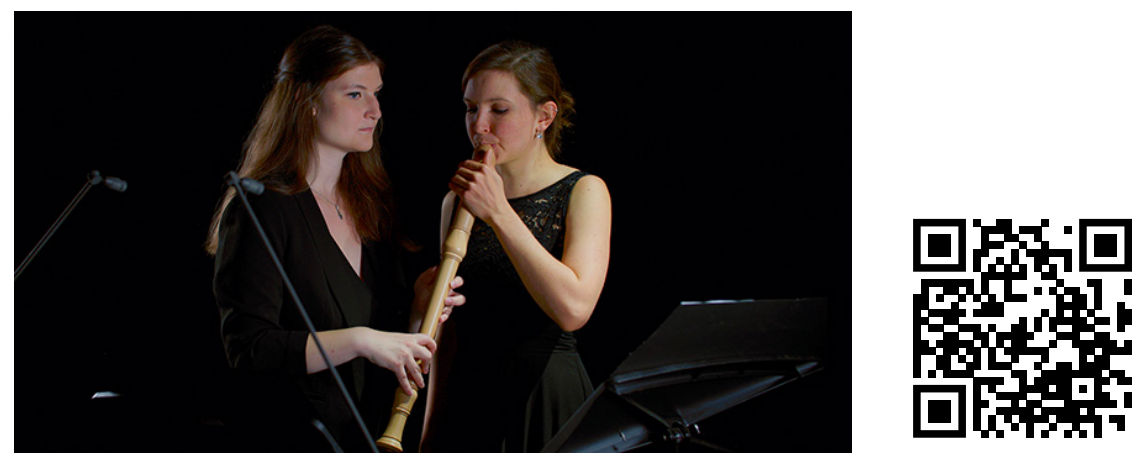

Abb. 59 Karin Wetzel: $N$ aus X-Pieces für Blockflöte, 2017. Lydia Dietrich und Laura Kalchofner, Blockflöte. Printed with permission. Zur Aufnahme: https://vimeo.com/640278455.
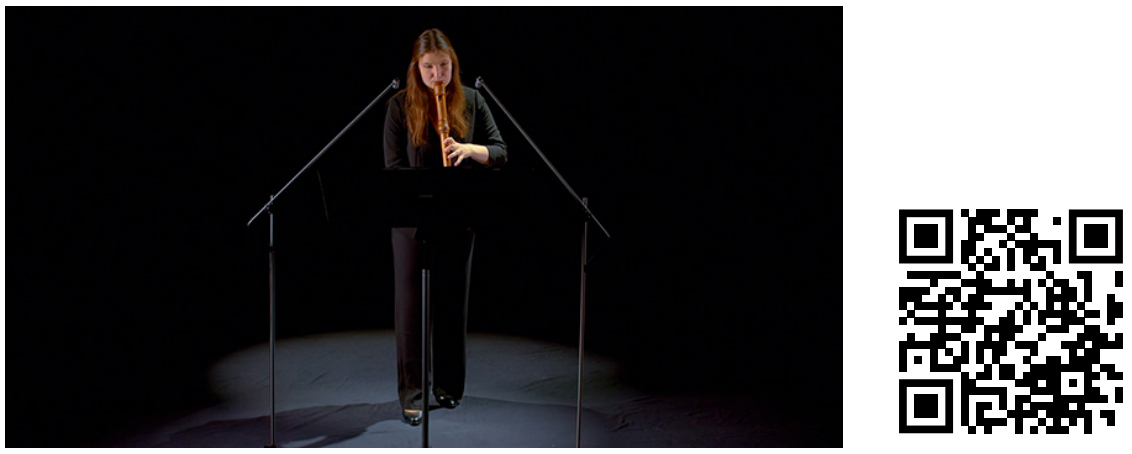

Abb. 6o Karin Wetzel: Backslash aus X-Pieces für Blockflöte, 2017. Laura Kalchofner, Blockflöte. Printed with permission. Zur Aufnahme: https://vimeo.com/640269631.

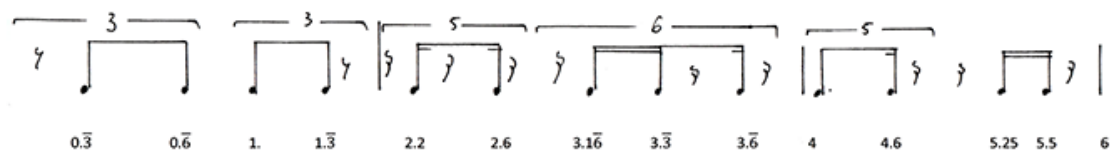

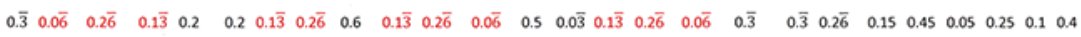

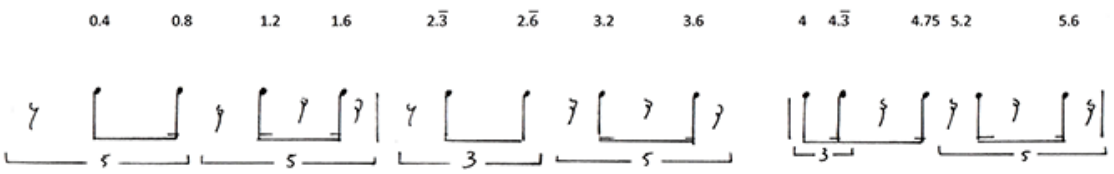

Abb. 61 Rhythmische Notenwerte und ihre numerischen Verhältnisse in Slash und Backslash, Takte 1-3. 
Der interformale Raum

Die beiden Solostücke Slash und Backslash als tragende Pfeiler der Komposition sind unabhängig voneinander aufführbar und haben ihren individuellen Verlauf. Auf einer rhythmischen Ebene sind sie aber auch sehr stark miteinander verschränkt. Um eine große rhythmische Vielfalt zu erhalten, habe ich im Vorfeld festgelegte Zahlenfolgen in Notenwerte übersetzt und folgendes Prinzip angewandt:

Abbildung 61 zeigt drei Zahlenreihen. Der Wert der Zahl 1 entspricht einer Viertelnote. Die obere Zahlenreihe entspricht dem Gerüstrhythmus ${ }^{1}$ der Artikulationsebene von Slash. Die untere Zahlenreihe entspricht dem Gerüstrhythmus der Artikulationsebene von Backslash. Die mittlere Reihe ist die Differenz zwischen den Werten der oberen und der unteren Zahlenreihe. Sie ist die Ausgangsreihe der Komposition. Das Ziel bestand darin, eine Ausgangsreihe $\mathrm{zu}$ entwickeln, in der identische Zahlensequenzen unterschiedliche rhythmische Texturen nach beiden Seiten generieren können. Bedingung für die Auswahl der Zahlen war, dass die Werte der resultierenden Zahlenreihen einem Notenwert entsprechen. Beispielsweise entspricht die Zahl o.4 dem zweiten Quintolensechzehntel einer Viertelnote, 1.2 dem zweiten Quintolensechzehntel der folgenden Viertelnoteneinheit usw. Gleiche Zahlenfolgen können beispielsweise auf die Instrumente neu verteilt werden. In Takt 2 generiert die Zahlenfolge $0.1 \overline{3}, 0.2 \overline{6}$, o.o $\overline{6}$ einen Quintolenrhythmus von 2.2. und 2.6 beziehungsweise 3.2 und 3.6 sowie einen dagegenstehenden Triolenrhythmus von $2 . \overline{3}$ und $2 . \overline{6}$ beziehungsweise $3 \cdot \overline{3}$ und $3 \cdot \overline{6}$. Gleiche Zahlenfolgen generieren hier zwar gleiche Rhythmen, werden aber über die Instrumente hinweg ausgetauscht. Identische Zahlenmuster können jedoch auch andere rhythmische Muster erzeugen, wenn sie innerhalb der Vierteleinheit beispielsweise unterschiedlich einrasten. Rastet die Zahlensequenz $0.1 \overline{3}, 0.2 \overline{6}, 0.0 \overline{6}$ einen Achtelwert später bei x.7 (statt x.2) ein, dann entstehen die rhythmischen Werte: $0.7,0.8 \overline{3}, 1.1,1.1 \overline{6}$. Die zeitlichen Intervalle zwischen den Impulsen sind in beiden Fällen identisch, die rhythmische Gestaltung wird jedoch weiter

1 Gerüstrhythmus bedeutet, dass die Zahlenwerte nur den Impulseinsatz darstellen, also nichts über die Dauer einer Note aussagen. Diese wurde frei von mir gestaltet und folgt keinem festgelegten Plan. Auch Zahlenketten mit sehr kurzen Notenwerten, kleiner als eine Sechzehntelnote, können frei in das Gerüst eingefügt werden. In diesem Fall zählt der Impulseinsatz der ersten Note der Kette. Die Länge der Notenkette und die Anzahl ihrer Notenwerte sind ebenfalls frei gestaltet. Auch Vorschläge sind frei zugefügt und unterliegen keinem rationalen System. An einigen Stellen habe ich mir Freiheiten genommen und bin vom Schema abgewichen. Vergleiche beispielsweise Takt 2, zweite Zählzeit von Backslash mit der Partitur. 
differenziert, denn statt in Triolen und Quintolen erfolgt die rhythmische Auflösung nun in Sextolen und Zehntolen. Durch die Verschiebungen identischer Zahlensequenzen innerhalb des Viertelrasters und immer neue Aufteilungen der rhythmischen Werte auf beide Instrumente entsteht eine große rhythmische Vielfalt, obwohl die zugrunde liegende numerische Abfolge in sich begrenzt und wiederholbar ist. Auch werden die beiden Stücke auf einer rhythmischen Ebene sehr eng miteinander verzahnt.

Die mittlere Ausgangszahlenkette, die an sich noch kein Rhythmus ist, hat folgende Form: ABA' CBC' AB'A' ABA'. ${ }^{2}$ Ich nenne diese Form eine interformale Brücke, die im Zwischenraum der Stücke existiert. Sie steuert lediglich die Verteilung der rhythmischen Impulse für beide Stücke auf der Artikulationsseite und ist dadurch als solche niemals für den Spieler oder Hörer wahrnehmbar.

Die rhythmische Ebene folgt sehr präzise gesetzten Parametern, kann von den Musikern in jeder Version kontrolliert werden und ist daher ein konstantes Merkmal des Stückes. Dagegen ist der Parameter Tonhöhe eine relative Variable und kein absolut gesetzter Wert. Er ist bereits in den Solofassungen schwer zu kontrollieren, deswegen könnte man ihn als emergent beschreiben. Da die Intonation und die resultierende Tonhöhe auf der Blockflöte von der Zusammenwirkung von Artikulation und Grifftechnik abhängig sind, kommt es durch die Teilung der Ebenen bereits in den Solofassungen - und mehr noch in den verschränkten Duofassungen mit ihrer physischen Desynchronisation zu unregelmäßigen und schwer vorhersehbaren Registerbrüchen in der Tonhöhenlinie.

Performance und Form

Artikulation und Greifen sind beim Spiel auf der Blockflöte zwei Prozesse, die normalerweise genau aufeinander abgestimmt sind. Die Desynchronisation von Artikulation und Grifftechnik bedeutet in den diagonalen Fassungen auch eine körperliche Trennung. Diese bringt verschiedene Konflikte mit sich. Beide Spieler finden sich in einer Situation wieder, in der ihnen die Kontrolle über Klang und Intonation permanent entgleitet und das Zusammenspiel zwischen Artikulation und Grifftechnik neu erschlossen werden muss. Gleichzeitig sind die Spieler als aktive Interpreten permanent dem Spiel des anderen Spielers passiv ausgesetzt. Erika Fischer-Lichte schreibt in ihrem Einführungsbuch zur Performativität:

2 Apostroph bedeutet, die Zahlenkette läuft rückwärts. 
„Wenn in performativen Prozessen die beteiligten Subjekte sowohl ihren Verlauf mitbestimmen als auch sich von ihm mitbestimmen lassen, ihnen agency sowohl verliehen als auch entzogen wird, erscheinen sie einerseits als aktiv Handelnde und zugleich andererseits als passiv die Wirkungen der Handlungen Anderer Erleidende." ${ }^{\text {3 }}$

Den aktiven Aspekt der Handlung und das passive Geschehenlassen sieht sie als zwei unterschiedliche Aspekte, die die Ambivalenz und die Unvorhersehbarkeit eines performativen Prozesses kennzeichnen und zu der Maxime führen, „daß das Handeln eine Bereitschaft einschließen solle, sich bestimmen zu lassen. ${ }^{4}$ Das in der westlichen Kultur als Gegensatz gedachte Begriffspaar "autonom" versus „fremdbestimmt" wird nach Fischer-Lichte in einer performativen Aufführung nicht als „Entweder-oder“, sondern als „Sowohl-alsauch“5 erlebt. Innerhalb des Werkzyklus findet also eine Transformation von einem autonomen zu einem heteronomen Spiel statt. Dadurch entwickelt sich das Stück weg von der Präsenz eines Konzertstückes, hin zu einer Performancesituation, die eine neue Form des Zusammenspiels erfordert und in der die Inszenierung des performativen, körperlichen Aktes in den Vordergrund rückt. Durch diesen Kniff kippt das Werk in einen anderen Zustand. Es kommt zu neuen Wechselwirkungen zwischen den einzelnen Faktoren, die auch bestimmte Konflikte aufwerfen. Auf der physischen Ebene erfordert die körperliche Verbindung beider Spieler einen neuen Zugang zu Spielpraxis, Technik, Haltung und Zusammenspiel. Auf der instrumentalen Ebene erfährt das Instrument Blockflöte eine Umdeutung. Die in sich gedrehte Flöte ist für den Spieler nicht mehr der vertraute Gegenstand. Die Blockflöten verbinden die Spieler physisch miteinander und mutieren durch die Drehung zu einem Knotenpunktim Spielernetzwerk. Auf derpsychologischen Ebene repräsentiert die Verschränkung eine extrem intime kammermusikalische Situation. Die Spieler spielen nicht nur gleichzeitig, sondern rücken auch räumlich in die denkbar dichteste Position zueinander. Beide Spielorte fallen zusammen und werden miteinander verschränkt. Aus diesem Grund müssen neue Wege der Kommunikation und Interaktion und vor allem eine neue Herangehensweise an das Proben gefunden werden. Bei den Proben zur Uraufführung des Stückes lautete daher die Kernfrage: Was probt man eigentlich in den verschränkten Versionen? Folgende Punkte schälten sich im Probenprozess heraus: Neben der Haltung - die Flöten müssen stabil und im Mund bleiben - ist es vor allem

3 Erika Fischer-Lichte: Performativität. Eine Einführung, Bielefeld: Transcript, 2013, S. 87.

4 Hans Ulrich Gumbrecht: „Die Emergenz der Emergenz“, in: Frankfurter Allgemeine Zeitung, Nr. 92, 19.4.2003, S. 38 .

5 Fischer-Lichte: Performativität, S. 68. 
die tatsächliche Trennung von Greifen und Artikulieren. Für die Solofassungen ist es noch vergleichsweise einfach, Finger und Artikulation zusammenzusetzen. Aber auf einer Flöte zu greifen, auf der man selbst nicht spielt, ist etwas komplett anderes und auch Irritierendes. Darüber hinaus musste eine Strategie des konsequenten Bei-sich-selbst-Bleibens entwickelt werden.

Gerade in Bezug auf den letzten Punkt zeigt sich eine Analogie zwischen der Performerebene und der Werkebene: Musiker, die sich für ein Ensemble zusammenfinden, sind Individuen. Außerhalb des Ensemblekontextes agieren sie als Personen unabhängig voneinander, verschmelzen aber idealerweise während der Aufführung zu einem Klangkörper. Werk und Ausführende haben beim Poly-Werk also die Merkmale Unabhängigkeit und Individualität sowie die Möglichkeit des Zusammenschlusses gemeinsam. Der Aspekt der Selbststabilisierung im Zusammenschluss als Grundlage für die Öffnung für das Gegenüber wird in X-Pieces sowohl auf der Werkebene als auch auf einer menschlich-performativen Ebene vollzogen.

\subsection{Komponieren in der Differenz}

Der künstlerische Anspruch in X-Pieces konzentrierte sich auf eine Entwicklung verschiedener Kombinationsformen, die über das rein schichtende und additive Prinzip hinausgehen und ebenso subtraktive und kreuzende Verfahren miteinbeziehen. Die Unterschreitung der bis dahin kleinsten Einheit einer Instrumentalstimme wird nicht nur auf das Werk, sondern ebenso auf den Körper des Musikers selbst übertragen. Die Zuordnung Solostück - Solist bildet nicht mehr die unterste Werk- und Instrumentalstufe, typischerweise gefolgt von der nächsten Stufe Duett - Duo. Der Rahmen differenziert sich weiter und erschließt ebenso dem Raum zwischen dem „Dividuum“ als einem in sich geteilten Wesen und der "Dyade“ als einer extrem engen und überindividuellen Duostruktur, sodass die Werkstufung jetzt Dividuum Individuum - Dyade - Duo lautet. Der schrittweise Transformationsprozess wird nicht durch ein rein additives Vorgehen erreicht, sondern durch eine Mischung additiver, subtraktiver, spaltender und kreuzender Verfahren sowohl auf den Ebenen des Werks als auch des Körpers. Eine eindeutige Addition ist dabei für die Zusammenführung der Solostücke Slash und Backslash zum Duo $V$ gegeben. Eine eindeutige Subtraktion findet in der Reduktion der Solostücke auf die nächstkleineren Soli Hidden Pieces statt. Die Soli Slash und Backslash bilden dabei selbst ein Poly-Werk, einen Schnittpunkt verschiedener Kreuzungen, in dem verschiedene Kontextualisierungsrichtungen angelegt sind. Das Hidden $V$ ist eine Addition subtrahierter Soli, die Version $X$ eine 
additive Kreuzung, in den Versionen $N$ geht die Addition beider Solostücke mit einer Spaltung und Kreuzung auf der Werk- und der Körperebene einher.

Meine beiden Solostücke Slash und Backslash würde ich als Zwillingswerke bezeichnen, die in ihrer Materialität und Rhythmik auf denselben Ursprung zurückgehen, denselben Grad an Unbestimmtheit aufweisen und identisch in der Instrumentation sind. Trotzdem sind sie einzeln aufführbar, besitzen ihre eigene Form, ihren eigenen Flow und ihre eigene charakteristische Physiognomie. Das Stück Backslash gewinnt seine Energie aus den kraftvollen Impulsen in $f$-bis ff-Dynamik, von denen sich die Linie immer wieder neu abstoßen kann (z. B. Takte 17-20, Takt 34, Takte 65-70, Takte 72-73). Dadurch ist es ein Stück weit perkussiverund rhythmischer als das SchwesterstückSlash, das sich aus dem wiederkehrenden Ausgangsmotiv (Takt 1, Takte 31/32, Takte 51/52, Takt 101) immer neu entwickelt. Die $V$-Version beider Stücke betont vor allem den divergierenden und kontrapunktischen Charakter zweier sich selbstständig bewegender Linien. Im Gegensatz zum traditionellen Kammermusikund Ensemblespiel soll die Aufführung nicht zwingend auf ein musikalisch genau aufeinander hörendes und perfekt abgestimmtes Zusammenspiel von zwei funktionalisierten Stimmen hinauslaufen. Die Spieler sollen sich vorrangig auf das eigene Spiel konzentrieren und sich des Spiels des anderen bewusst sein. Das soll aber nicht ausschließen, dass die Spieler den Kontakt zueinander suchen können und abschnittsweise zusammen musizieren. Die Entscheidung darüber soll individuell, spontan und zufällig erfolgen. Je nach Raum und räumlichem Abstand wird bei einem kleinen räumlichen Intervall eher ein linear-kontrapunktischer Effekt der Zweistimmigkeit, bei einer entfernten Vergleichzeitigung idealerweise ein räumlicher Moiréeffekt erzeugt. Die Versionen $N$ und $X$ stellen die physische Desynchronisation der Spielaktionen und die körperliche Neuverbindung der Spieler in den Vordergrund. Sie teilen sich die Flöten, rücken körperlich sehr eng zusammen und erleben eine Verschmelzung zu einer gebrochenen Einheit.

Die typische Zusammensetzung eines Poly-Werks basiert immer auf der Bestimmung kleinster integraler Werkeinheiten - bestehend aus einer oder mehreren Stimmen -, die eine Werkidentität bilden. Ihnen können weitere Werkeinheiten hinzugefügt werden, die eine Neukontextualisierung der Werkidentität ermöglichen. Die unterste Möglichkeit einer kleinsten, unteilbaren Einheit bildete dabei normalerweise das Solo. In X-Pieces ist auch dieses teilbar und trägt als Solo-Poly-Werk eine Differenz in sich. Die ausgeschriebene Artikulationsstimme erzeugt in ihrer Interaktion mit der - auch als ganzwertig aufzufassenden - Fingerstimme eine völlig neue Kontextualisierung derselben. Das zeigt, dass die Gattung Poly-Werk nicht nur in der Lage ist, eine Öffnung und Neukontextualisierung von Werkidentität im Zusammenhang 
zwischen Werkindividuum und Werkkollektiv zu vollziehen, sondern diese Kontextualisierungsrichtung auch umzukehren. Während die additive Entstehungsrichtung immer eine Wandlung und Neusituierung der Werkidentität in verschiedenen kollektiven Kontexten ist, stellt die subtraktive Entstehungsrichtung eine Befragung der kollektiven Kontexte im Einzelnen dar. In X-Pieces werden ausgehend von den Soli Slash und Backslash beide Richtungen verfolgt und zusätzlich noch miteinander kombiniert. Meine Ausgangsfrage nach der Herausforderung der Identität im Verschiedenen kehrte sich letztlich um zu einer Befragung des Verschiedenen innerhalb einer Identität. Die Praxis des „Komponierens in der Differenz" ist dabei der eigentliche Schlüssel, um die verschiedenen Kontexte vom Moment ihrer Entstehung an so ineinander zu verknüpfen, dass die möglichen Versionen in jeder einzelnen Version von Anfang an präsent sind. Auf diese Weise wird die Erweiterung und Komplementierung der individuellen Werkidentität zu einer sozialen Werkidentität bereits im Einzelnen selbst vollzogen. 